\title{
REMOCIÓN DE CROMO HEXAVALENTE SOBRE RESIDUOS DE CACAO PRETRATADOS QUÍMICAMENTE
}

\section{REMOVING OF HEXAVALENT CHROMIUM ON CHEMICALLY PRETREATED CACAO WASTE}

\author{
Candelaria Tejada Tovar ${ }^{1 *}$, Ángel Villabona Ortiz $^{2}$, María Jiménez Villadiego ${ }^{3}$
}

\begin{abstract}
${ }^{1}$ Magíster en Ingeniería Ambiental, Magíster en Educación, Especialista en Química Analítica, Ingeniera Química, Profesora de tiempo completo del Programa de Ingeniería Química y docente del grupo de investigación IDAB de Ingeniería. Universidad de Cartagena, e-mail: ctejadat@unicartagena.edu.co; ${ }^{2}$ Magíster en Ingeniería Ambiental, Especialista en Ingeniería Sanitaria y Ambiental, Profesor titular de tiempo completo del Programa de Ingeniería Química y docente del grupo de investigación IDAB. Universidad de Cartagena, e-mail: avillabonao@unicartagena.edu.co; ${ }^{3}$ Ingeniera Química, e-mail: MJimenez_Ingqca@hotmail.com; *Investigador para correspondencia.
\end{abstract}

Rev. U.D.C.A Act. \& Div. Cient. 20(1): 139-147, Enero-Junio, 2017

\section{RESUMEN}

La disponibilidad de recurso hídrico de calidad es un factor de gran interés científico, debido a la gran carga de contaminantes de origen antrópico presente en las fuentes de agua, siendo los metales, de especial atención por la bioacumulación y lamtoxicidad. En el presente artículo, se estudia la adsorción de Cromo hexavalente, usando cáscara de cacao, como material adsorbente, así como dos tratamientos químicos a la misma, con hidróxido de sodio y ácido clorhídrico, evaluando su efecto en la remoción del ión metálico. La medición del metal en solución, se hizo mediante espectroscopia UV-Vis, usando el reactivo difenilcarbazida. Se estudió el efecto del tamaño de partícula en el proceso y el ajuste de la cinética, se hizo con los modelos de primer orden, segundo orden, Elovich y difusión. Se encontró que el modelo que mejor se ajustó a los datos experimentales fue el de Elovich, mientras que la isoterma de Freundlich describe mejor el proceso de adsorción; de este modo, se establece que la superficie catalítica del bioadsorbente es heterogénea, exhibiendo diferentes energías de activación. Además, se observó que la modificación con ácido clorhídrico mejoró la capacidad de adsorción del material.

Palabras clave: Adsorción, biomaterial, Cromo (VI), tratamiento químico.

\section{SUMMARY}

The availability of quality water resources is a factor of great scientific interest because of the high burden of anthropic contaminants in water sources, with particular attention to metals due to their bioaccumulation and toxicity. In this research the adsorption of hexavalent chromium using cocoa shell as adsorbent material, as well as two chemical treatments with sodium hydroxide and hydrochloric acid was studied. The effect of removing this metal-ion was evaluated. Measurement of this metal in aqueous solution was realized by UV-Vis spectroscopy using diphenylcarbazide reagent. The effect of particle size in the process was studied and the adjustment of the kinetics was done with models of first order, second order, Elovich and diffusion It was found that the model with the best fit to the experimental data was Elovich, while Freundlich's isotherm describes the adsorption process in a better way. Thereby it was established that the catalytic surface of this bioadsorbent is heterogeneous, exhibiting different energies of activation. Furthermore it was observed that the modification with hydrochloric acid improved the adsorption capacity of this material.

Key words: Adsorption, biomaterial, Chromium (VI), chemical treatment.

\section{INTRODUCCIÓN}

La disponibilidad de agua potable de calidad es un factor importante para preservar la salud de la población; el creciente desarrollo de la sociedad hace aumentar, continuamente, la cantidad y los tipos de fuentes de contaminación ambiental $\mathrm{y}$, así, numerosos efluentes industriales, plaguicidas y otros productos químicos utilizados en la agricultura, arrastrados por las lluvias, contribuyen al deterioro de la calidad de las aguas, siendo los metales pesados, de especial cuidado, por su alta toxicidad y alta permanencia en el ambiente (Islam et al. 2015). 
El cromo, se ubica como uno de los elementos más contaminantes, además de no ser química ni biológicamente degradable, por tanto, puede permanecer indefinidamente en el medio ambiente. Este metal presenta dos estados de oxidación: $\mathrm{Cr}$ (III) y $\mathrm{Cr}$ (VI). El primero de estos, aunque no es de carácter tóxico, mediante un proceso de óxido-reducción, se puede convertir en $\mathrm{Cr}(\mathrm{VI})$, el cual, es considerado tóxico, por ser capaz de absorberse, a través de la piel, provocando efectos negativos en la salud (Ma et al. 2015; Lavado-Meza et al. 2012).

Las descargas de $\mathrm{Cr}(\mathrm{VI})$ en aguas residuales provienen, generalmente, de curtiembres, de industrias metalúrgicas y maderas, además, de fertilizantes artificiales, los cuales, superan ampliamente la concentración máxima establecida en agua potable, por la Environmental Protection Agency (EPA), de $100 \mu \mathrm{g} / \mathrm{L}$ (Mädler et al. 2016). Se han usado diversas tecnologías para la remoción de este iones metálicos en aguas residuales, entre las que destacan: precipitación química, intercambio iónico, membranas, extracción con solventes, osmosis inversa, entre otras; sin embargo, muchas son ineficientes, además de involucrar un gran uso de químicos, generando grandes cantidades de residuos contaminantes (Torab-Mostaedi et al. 2013).

La adsorción ha sido estudiada como un método alternativo a las tecnologías convencionales para remover metales pesados en soluciones acuosas, que involucra la separación de una sustancia presente en una fase fluida, en la superficie de un adsorbente sólido. El proceso ofrece flexibilidad en el diseño y operación y produce un efluente tratado de alta calidad, siendo el carbón activado el material más usado en los procesos de adsorción (Asasian et al. 2014). Su utilidad deriva en la gran cantidad de microporos y mesoporos que resulta en la alta área superficial (Guo et al. 2016; Ingole \& Dharpal, 2012); sin embargo, el alto costo de su activación, encarece su uso en plantas de tratamientos (Largitte et al. 2016). Así ha ganado importancia la bioadsorción, usando adsorbentes de origen orgánico, como residuos agrícolas, microorganismos y biomasa de algas, para el tratamiento de aguas residuales, ya que es posible el uso de materiales más económicos, con igual eficiencia. La eficiencia del proceso, se basa en la presencia de grupos funcionales en la superficie de estos biomateriales, afines por los iones metálicos; estos grupos incluyen carboxilo, hidroxilo, sulfoxidos y amida (Feizi \& Jalali, 2015; Guo et al. 2016).

Entre los materiales residuales ampliamente estudiados y con excelentes resultados para la remoción de metales pesados, se encuentran: cáscaras de naranja (Tejada et al. 2015a), aserrín de pino (Momčilović et al. 2011), bagazo de palma (Ismaiel et al. 2013; Tejada et al. 2015b), bagazo de caña (Hamza et al. 2013), cáscara de nuez (Altun \& Pehlivan, 2012), cáscaras de yuca y de limón (Tejada et al. 2015c), entre otros.
La cáscara de cacao ha sido identificada como un sorbente natural muy eficiente para la remoción de iones plomo y otros metales pesados, mostrando ser eficiente frente a otro tipo de materiales residuales (Wu et al. 2013).

En el presente trabajo, se estudió la capacidad de adsorción de Cr (VI) usando, como biomasa residual, cáscara de cacao. Los ensayos de adsorción, se realizaron en sistemas por lotes, estudiando el efecto del biomaterial modificado químicamente, con hidróxido de sodio y ácido clorhídrico, en la adsorción del ión metálico en estudio.

\section{MATERIALES Y MÉTODOS}

Materiales. Los agentes químicos usados en el presente estudio fueron grado analítico marca Merck. Además, para todos los análisis, se utilizó agua desionizada. La cáscara de cacao fue obtenida de cultivos de cacao, ubicados en el municipio de San Jacinto, departamento de Bolívar (Colombia).

Preparación del adsorbente. Las cáscaras fueron lavadas con agua destilada, con el fin de retirar impurezas solubles y suciedad. Luego, fueron secadas por 24 horas en horno, a una temperatura de $90^{\circ} \mathrm{C}$, reducidas de tamaño y tamizadas, seleccionando los tamaños de partícula inferiores a 0,355mm. Una vez preparadas, se procedió a la caracterización físicoquímica de las mismas, realizando una determinación de elementos CHON (Carbono: AOAC 949.14; Hidrógeno: AOAC 949.14; Nitrógeno: AOA 984.13; Azufre: Digestión), además de pectina, lignina, celulosa, hemicelulosa (Digestión-termogravimetría). Igualmente, se realizó un análisis de los grupos funcionales presentes en la biomasa antes y después de la adsorción, utilizando un espectrómetro infrarrojo por transformada de Fourier, en condiciones ambientales. Los espectros, se registraron a partir de 4000 a $400 \mathrm{~cm}^{-1}$.

Modificación química. La biomasa fue tratada con hidróxido de sodio y ácido clorhídrico $1 \mathrm{M}$; para tales efectos, fueron puestos en contacto $20 \mathrm{~g}$ de biomasa en $100 \mathrm{~mL}$ de solución, a temperatura ambiente $\left(30^{\circ} \mathrm{C}\right)$, durante 24 horas, a una agitación constante de 150rpm. Alcanzado el tiempo de contacto, se realizó un repetido lavado con agua destilada, hasta obtener un valor constante de $\mathrm{pH}$. Luego, la biomasa se secó por 12 horas, en horno, a $70^{\circ} \mathrm{C}$ (Liu et al. 2014; Abdolali et al. 2015; Chieng et al. 2015).

Preparación de soluciones y determinación de Cr (VI). Las soluciones de agua sintética residual fueron preparadas con el reactivo $\mathrm{K}_{2} \mathrm{Cr}_{2} \mathrm{O}_{7}$ (dicromato de potasio), a una concentración de 100ppm. Un método espectrofotométrico fue usado para determinar la cantidad de $\mathrm{Cr}(\mathrm{VI})$ removido. El complejo rosado formado por la reacción entre el ion $\mathrm{Cr}(\mathrm{VI})$ y la 1,5-difenilcarbazida fue medido, a 540nm, usando agua destilada, como blanco, en el espectrofotómetro Uv-Vis (Wu et al. 2013). 
Experimentos de adsorción en sistema por lotes. En el estudio, para determinar el mejor tamaño de partícula, fue llevado a cabo a temperatura de $\left(25^{\circ} \mathrm{C}\right)$, por 120 minutos, a pH 2 y la relación biomasa/solución fue de $5 \mathrm{~g} / \mathrm{L}$. El efecto del tamaño de partícula $(0,355-0,5-1 \mathrm{~mm})$ fue medido, a una concentración inicial del metal de 100 ppm. El pH de la solución fue ajustado por adición de $\mathrm{HCl}$ o $\mathrm{NaOH} 1 \mathrm{M}$. Por último, se hizo el estudio cinético por 270 minutos, tanto para la biomasa modificada como sin modificar. Los experimentos fueron realizados al menos por duplicado, bajo iguales condiciones.

\section{RESULTADOS Y DISCUSIÓN}

Caracterización físico-química de la cáscara de cacao. En la tabla 1 , se relacionan los métodos analíticos y los parámetros utilizados, para determinar la composición elemental de las cáscaras de cacao. De Los resultados obtenidos, se establece que el carbono es el elemento con mayor porcentaje. Además, se confirma la presencia de lignina, de celulosa y de hemicelulosa, por lo cual, se esperaría una alta eficiencia de adsorción, puesto que todos estos grupos hidroxilos y fenólicos pueden favorecer la adsorción de metales (Chen et al. 2012).

Tabla 1. Caracterización de la cáscara de cacao.

\begin{tabular}{|c|c|c|}
\hline Parámetros & Valor & Métodos \\
\hline Carbono \% & 50,35 & AOAC 949.14 \\
\hline Hidrógeno \% & 5,08 & AOAC 984.14 \\
\hline Nitrógeno \% & 1,28 & AOAC 984.13 KJELDAHL \\
\hline Azufre ppm & 0,59 & Digestión-nefelometría \\
\hline Cenizas \% & 7,75 & Termogravimetría \\
\hline Pectina \% & 9,54 & Digestión ácida-termogravimetría \\
\hline Lignina \% & 12,66 & Fotocolorimetría \\
\hline Celulosa \% & 19,82 & Digestión-termogravimetría \\
\hline Hemicelulosa \% & 9,45 & Digestión-termogravimetría \\
\hline Calcio mg/g como $\mathrm{Ca}^{2+}$ & 11,20 & EAA \\
\hline Sodio mg/g como $\mathrm{Na}^{+}$ & 0,5 & EAA \\
\hline Potasio mg/g como $\mathrm{K}^{+}$ & 47 & EAA \\
\hline Hierro mg/g como $\mathrm{Fe}^{2+}$ & 0,0014 & EAA \\
\hline Cobre $\mathrm{mg} / \mathrm{g}$ como $\mathrm{Cu}^{2+}$ & 0,008 & EAA \\
\hline Magnesio mg/g como $\mathrm{Mg}^{2+}$ & 2,20 & EAA \\
\hline Cromo mg/g como $\mathrm{Cr}^{3+}$ & 0,0006 & EAA-Horno de grafito \\
\hline
\end{tabular}

Experimentos de adsorción. De los resultados mostrados en la figura 1 , se encuentra que es el tamaño de $0,355 \mathrm{~mm}$, el cual, favorece el proceso de adsorción, así se establece que, a un menor tamaño, se podría dar un aumento en el área superficial; sin embargo, se sugiere realizar estudios de caracterización de poros a la biomasa. Tal como lo reporta la literatura, el aumento de microporos aumenta el número de sitios accesibles, pero no puede ser relacionado con el tamaño de partícula, pues en realidad, se están usando partículas con un mismo espesor, que es lo que determina la distancia de difusión y la clasificación de tamaño de la partícula de una biomasa por tamices estándar, que funciona en longitud y ancho (Igwe \& Abia, 2006). Sala et al. (2005) mostraron que las partículas de biomasa más grandes de Sargassum fluitans y Ascophylum nodosum tenían mayor adsorción de metal que las partículas más pequeñas, al aumentar de $0,15 \mathrm{~mm}$ a $0,85 \mathrm{~mm}$, pero a partir del tamaño de partícula $0,85 \mathrm{~mm}$ a $1,00 \mathrm{~mm}$, la cantidad adsorbida disminuía.

Equilibrio de adsorción de la cáscara de cacao y sus modificaciones. Si se analiza la cinética del proceso, se establece que todos los biomateriales superan el $80 \%$ de adsorción, luego de los 290 minutos, durante los cuales, se estudió la remoción del ión metálico. De la figura 2, se observa, además, que la modificación con $\mathrm{NaOH}$ no supera en ningún momento los porcentajes alcanzados por la biomasa sin modificación y, también, que la hecha con $\mathrm{HCl}$ solo lo hace ligeramente; sin embargo, solo con el estudio isotérmico a 


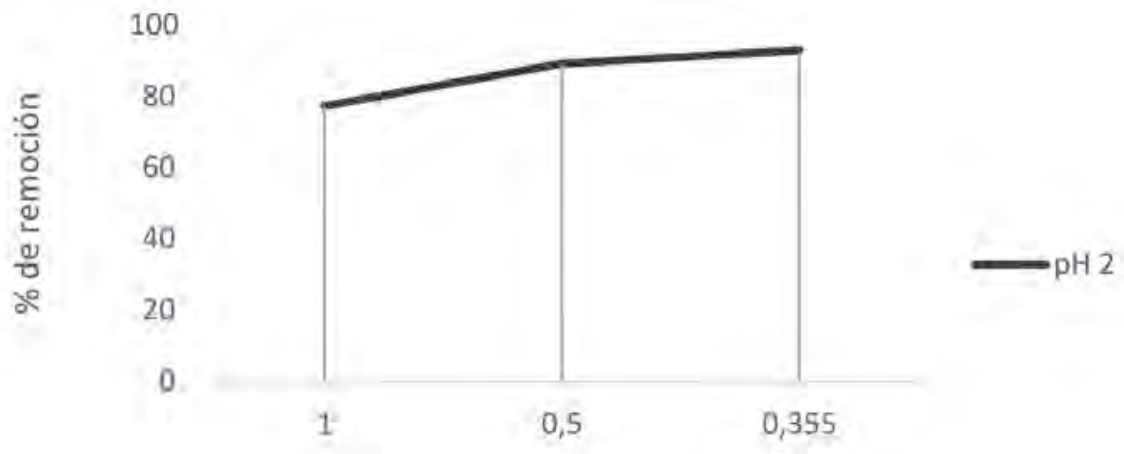

Tamaño de partícula

Figura 1. Efecto del tamaño de partícula en la adsorción de Cr (VI).

diferentes concentraciones iniciales de metal, se determinará la capacidad máxima de cada biomaterial.

Al realizar el ajuste de los modelos cinéticos a los datos experimentales, tal como se observa en las figuras 3(a), (b) y (c), se establece que el modelo cinético de Elovich es el que se ajusta de mejor forma a los datos experimentales del proceso de adsorción. Esto indica, que el mecanismo controlante de la adsorción sobre la cáscara de cacao es una reacción de segundo orden y, además, la superficie catalítica de la misma es heterogénea, exhibiendo diferentes energías de activación. En este modelo, $\alpha$ se relaciona con la energía de quimisorción y $\beta$, con la superficie cubierta

El análisis de isotermas de adsorción estuvo basado en el análisis de los parámetros calculados, mostrados en la tabla
2, pudiendo establecer que la máxima capacidad de adsorción es alcanzada con las cáscaras de cacao modificadas con ácido clorhídrico. Asimismo, se observa que los valores de la velocidad inicial de adsorción son mayores en la cáscara de cacao sin modificar, al igual que $\beta$, lo que muestra una toma más rápida por la biomasa sin modificar, aunque esto no signifique que sea la de mayor eficiencia.

Respecto al análisis de isotermas de adsorción, se establece la correlación de los datos experimentales con el modelo de Freundlich, así se puede concluir que el enlace se da, principalmente, por fuerzas físicas; la adsorción está limitada a la formación de una monocapa; el número de especies adsorbidas no excede el total de sitios disponibles y no se considera ninguna reacción de desorción que pueda tener lugar durante el proceso de remoción (Alves \& Gil, 2009).

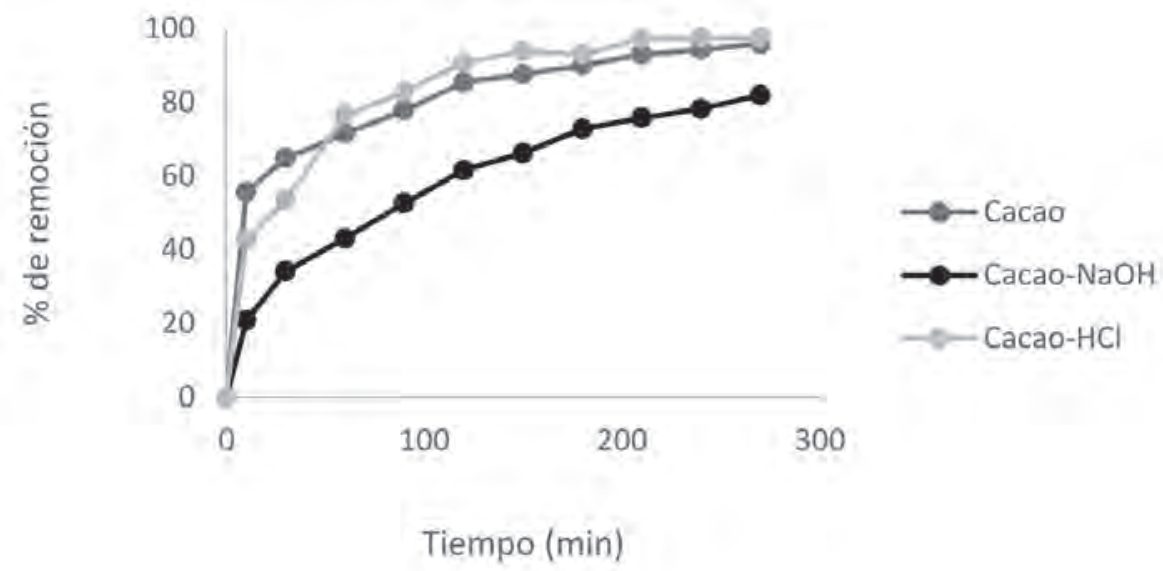

Figura 2. Equilibrio de la adsorción de Cr (VI) sobre cáscara de cacao. 


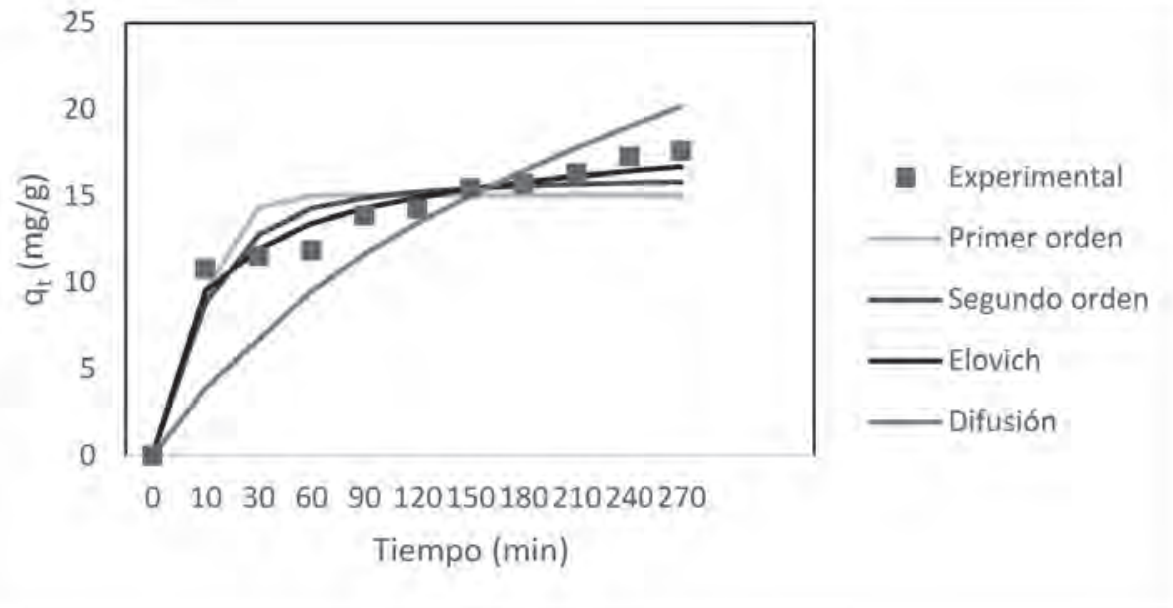

(a)

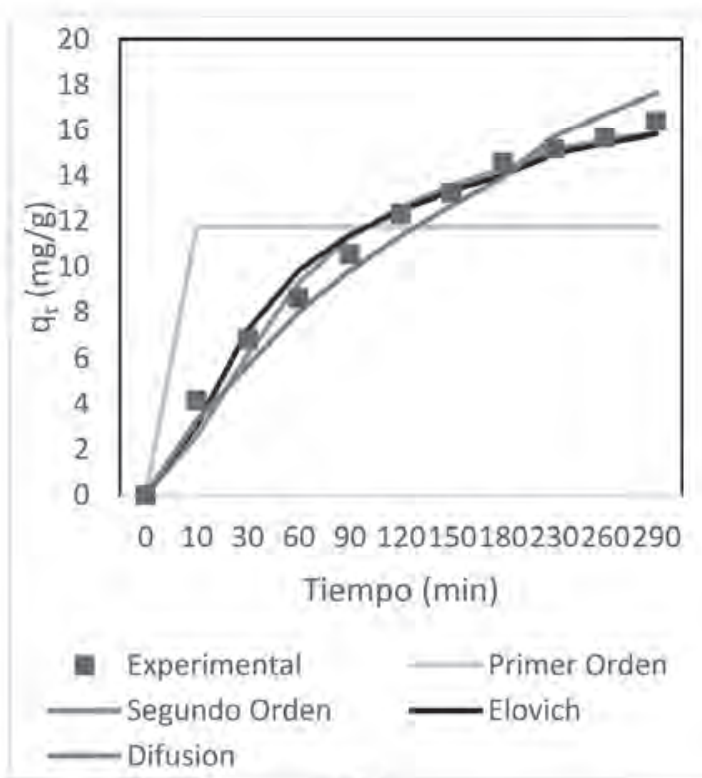

(b)

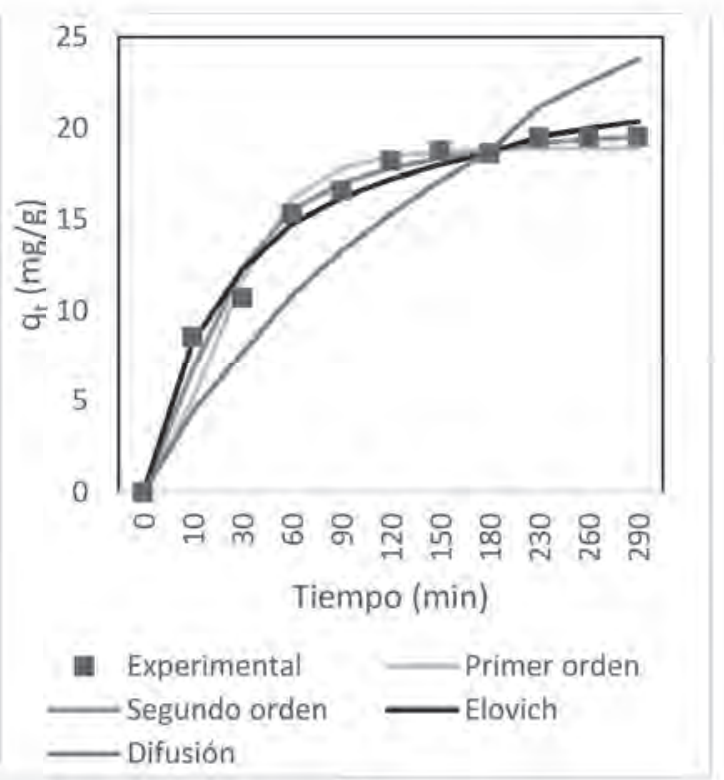

(c)

Figura 3. Ajuste de modelos cinéticos $\mathrm{Cr}$ (VI). (a) cáscara de cacao sin modificar, (b) modificada con $\mathrm{NaOH}$ y (c) modificada con $\mathrm{HCl}$.

Tabla 2. Parámetros de modelos de isotermas para la adsorción de $\mathrm{Cr}(\mathrm{VI})$.

\begin{tabular}{|c|c|c|c|c|}
\hline Modelo & Parámetros & $\begin{array}{c}\text { Cáscara de } \\
\text { cacao }\end{array}$ & $\begin{array}{c}\text { Cáscara de cacao } \\
\text { modificada con NaOH }\end{array}$ & $\begin{array}{c}\text { Cáscara de cacao } \\
\text { modificada con HCl }\end{array}$ \\
\hline \multirow{3}{*}{ Langmuir } & $\mathrm{qmax}$ & 227,3484 & 76,4416 & 463,5226 \\
\cline { 2 - 5 } & $\mathrm{b}$ & 0,0112 & 0,0019 & 0,0018 \\
\cline { 2 - 5 } & $\mathrm{suma}$ & 13,4484 & 34,6255 & 8,6184 \\
\hline \multirow{3}{*}{ Freundlich } & $\mathrm{Kf}$ & 0,9571 & $2,84 \mathrm{E}-05$ & 0,3863 \\
\cline { 2 - 5 } & $1 / \mathrm{n}$ & 1,5336 & 3,2730 & 1,2897 \\
\cline { 2 - 5 } & $\mathrm{suma}$ & 0,5782 & 8,9567 & 5,8334 \\
\hline
\end{tabular}


De la caracterización a la biomasa, se observa que es el carbono el elemento con mayor porcentaje; además, se confirma la presencia de lignina, de celulosa y de hemicelulosa, que son bien conocidos por la presencia de grupos funcionales hidroxilos y carboxilos y que han sido relacionados con la remoción de metales. Se establece que es el modelo de Elovich el que describe, de mejor forma, el proceso de adsorción, por lo que se sugiere que la superficie catalítica del bioadsorbente es heterogénea, exhibiendo diferentes energías de activación. De acuerdo a la isoterma de Langmuir, la máxima capacidad de adsorción se encontró con la cáscara de cacao modificada con $\mathrm{HCl}$; además, se mostró que la modificación con $\mathrm{NaOH}$ disminuye la capacidad de la biomasa.

Se comparó la capacidad de adsorción de adsorbentes, previamente reportados para $\mathrm{Cr}(\mathrm{VI})$, con los presentes resultados, de lo que se estableció, que la cáscara de cacao es una mejor alternativa frente a otros adsorbentes de bajo costo, pues su modificación con $\mathrm{HCl}$ alcanza una capacidad de $463 \mathrm{mg} / \mathrm{g}$, en comparación con 250mg/g-cáscara de mosambi (Citrus limetta) (Saha et al. 2013), 278mg/g-cáscara de arroz modificada (El-Shafey, 2005), 131mg/g-cáscara de maní modificada (Owalude \& Tella, 2016), 0,582mg/g-Kenaf (Hibiscus cannabinus) (Omidvar et al. 2016), 9mg/g-carbón de cáscaras de coco (Babel \& Kurniawan, 2004), 69,5mg/gpino de chipre (Pinus brutia) (Ozdes et al. 2014), entre otros.

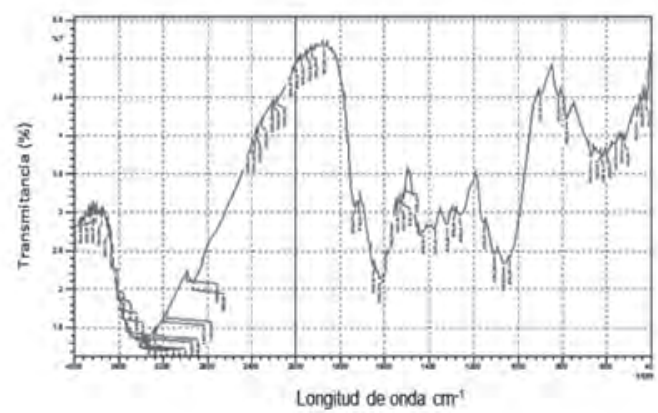

(a)

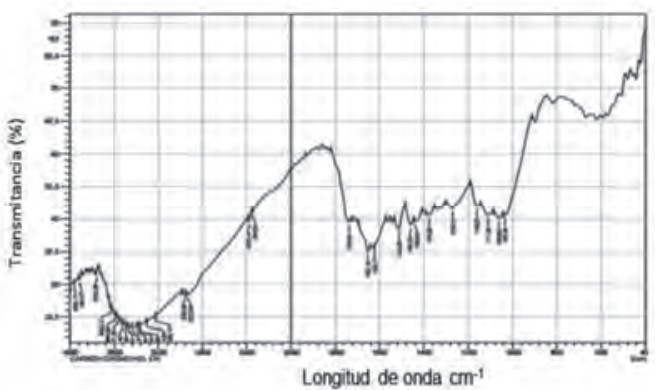

(c)
Asimismo, con el análisis de los espectros FTIR fue posible identificar los grupos funcionales de la biomasa de cacao, relacionados con la adsorción de metales pesados y la eficiencia de la misma.

En la figura 4 (a), correspondiente a la cáscara de cacao antes de la adsorción, la región desde 3700 a $2900 \mathrm{~cm}^{-1}$ concierne a la vibración por extensión del hidrógeno y un átomo de oxígeno, lo que favorece la aparición de picos de adsorción; además, se observa una tendencia decreciente en este rango y se debe a que el enlace de hidrógeno tiende a ampliar los picos y desplazarlos hacia números de onda más bajos, por tanto, la forma ancha y de gran intensidad del pico sobre 3300 $\mathrm{cm}^{-1}$ es típica (Amel et al. 2012). La vibración de enlaces C-H, correspondiente a grupos alifáticos, se encontró en la región comprendida entre $2990-2800 \mathrm{~cm}^{-1}$. Asimismo, la tendencia creciente del espectro lo genera la formación de triples enlaces, que se favorecen en la región de 2700 a $1850 \mathrm{~cm}^{-1}$; para el caso en estudio, los enlaces de este tipo corresponden a grupos funcionales asociados al nitrógeno, donde se observa la aparición de grupos del tipo $\mathrm{C} \equiv \mathrm{N}$ sobre $2374 \mathrm{~cm}^{-1}$, del mismo modo, la aparición de $\mathrm{C} \equiv \mathrm{C}$, sobre la región de $2026 \mathrm{~cm}^{-1}$. La región del pico sobre 1950 a $1550 \mathrm{~cm}^{-1}$ pertenece a los enlaces dobles, $\mathrm{C}=\mathrm{C}$ y $\mathrm{C}=\mathrm{O}$, correspondiente a grupos aromáticos y grupo carbonilo de éster, respectivamente. En general, se estableció que en la biomasa analizada hay mayor presencia de hidrocarburos alifáticos, aromáticos e insaturados, con una energía vibracional variable.

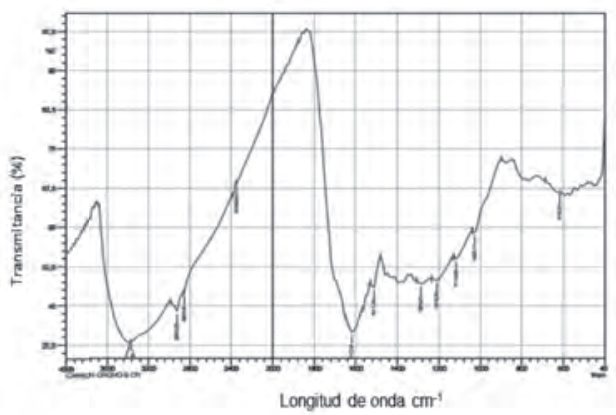

(b)

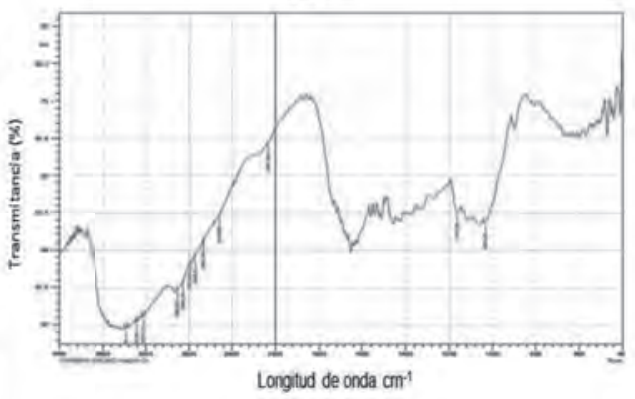

(d)

Figura 4. Análisis FTIR. (a) Cáscara de cacao antes de la adsorción, (b) cáscara de cacao-Cromo, (c) cáscara de cacao HCl y (d) cáscara de cacao $\mathrm{NaOH}$. 
Al comparar con los grupos funcionales que aparecen luego de la adsorción de cromo, tal como se observa en la figura 4(b), se encontró que hay un cambio en los picos a 1800 y $1600 \mathrm{~cm}^{-1}$, correspondiente a la vibración $\mathrm{C}=\mathrm{C}$. Además, el pico a $1440 \mathrm{~cm}^{-1}$, asociado al estiramiento del anillo aromático, también sufre un cambio, al igual que los ubicados a 1240 y $1080 \mathrm{~cm}^{-1}$, relacionados con los enlaces C-H y C-O. En los espectros de la cáscara de cacao tratada [Figuras 4(c) y (d)], se da un cambio en las bandas, entre 3600 y $3200 \mathrm{~cm}^{-}$ ${ }^{1}$, correspondiente a los grupos - $\mathrm{OH}$ y, a diferencia de la modificación con $\mathrm{NaOH}$, en la modificación hecha con $\mathrm{HCl}$, se observa un cambio en el pico que aparece a $1500 \mathrm{~cm}^{-1}$, correspondiente a la vibración $\mathrm{C}=\mathrm{C}$. Así se establece que este grupo puede ser importante en la remoción del cromo y, de allí, la diferencia significativa entre la capacidad de adsorción de cada material.

En conclusión, se observó que la biomasa cuenta con grupos hidroxilo, los cuales, se han encontrado en la mayoría de los carbones activados, utilizados como producto principal en la remoción de contaminantes y se establece, que son principalmente estos grupos los responsables de la toma metálica (Ahmad et al. 2012); sin embargo, cabe destacar, que los grupos funcionales que contienen $\mathrm{N}$ y $\mathrm{S}$ también pueden ser importantes en la adsorción de metales, quizás diferentes al cromo, dependiendo de factores, como el estado químico, la accesibilidad, la cantidad de sitios activos y la afinidad entre los sitios y el metal.

Agradecimientos: Los autores desean agradecer al Departamento de Ciencias, Tecnologías e Innovación de Colombia COLCIENCIAS y a la Universidad de Cartagena, por la financiación de este proyecto, en el marco del programa Jóvenes Investigadores e Innovadores. Conflicto de intereses: El manuscrito fue preparado y revisado con la participación de todos los autores, quienes declaramos que no existen conflicto de intereses que ponga en riesgo la validez de los resultados presentados.

\section{BIBLIOGRAFÍA}

1. ABDOLALI, A.; NGO, H.H.; GUO, W.; ZHOU, J.; DU, B.; WEI, Q.; WANG, X.C.; DAN NGUYEN, P; 2015. Characterization of a multi-metal bindingbiosorbent: Chemical modification and desorption studies. Biores. Technol. 193:477-487.

2. AHMAD, F.; ASHRI, W.; RADZI, R. 2012. Cocoa (Theobroma cacao) shell-based activated carbon by $\mathrm{CO}_{2}$ activation in removing of Cationic dye from aqueous solution: Kinetics and equilibrium studies. Chem. Eng. Res. Design. 90(10):1480-1490.
3. AltUN, T.; PEHLIVAN, E. 2012; Removal of Cr(VI) from aqueous solutions by modified walnut shells. Food Chemistry. 132:693-700.

4. ALVES, L.V.; GIL, L.F. 2009; Adsorption of Cu (II), Cd(II) and $\mathrm{Pb}$ (II) from aqueous single metal solutions by succiny lated twice-mercerized sugarcane bagasse functionalized with tri ethylen etetramine. Water Research. 3(18):4479-4488.

5. AMEL, K.; ABDESLAM, M.; KERROUM, D. 2012. Isotherm and kinetics study of biosorption of cationic dye onto banana peel. Energy Procedia. 19:286295.

6. ASASIAN, N.; KAGHAZCHI, T.; FARAMARZI, A.; HAKIMISIBONI, A.; ASADI-KESHEH, R.; KAVAND, M.; MOHTASHAMI, S. 2014. Enhanced mercury adsorption capacity by sulfurization of activated carbon with SO2 in a bubbling fluidized bed reactor. J. Taiwan Inst. Chem. Eng. 45(4):1588-1596.

7. BABEL, T.; KURNIAWAN, A. 2004. Cr(VI) removal from synthetic wastewater using coconut shell charcoal and commercial activated carbon modified with oxidizing agents and/or chitosan. Chemosphere, 54:951-967.

8. CHEN, G.; FAN, J.; LIU, R.; ZENG, G.; CHEN, A.; ZOU, Z. 2012. Removal of Cd (II), Cu (II) and Zn (II) from aqueous solutions by Phanerochaete chrysosporium. Environ. Technol. 33(23):2653-2659.

9. CHIENG, H.I.; LIM, L.B.L.; PRIYANTHA, N. 2015. Enhancing adsorption capacity of toxic malachite green dye through chemically modified breadnut peel: equilibrium, thermodynamics, kinetics and regeneration studies. Environ. Technol. 36(1):86-97.

10. EL-SHAFEY, E. 2005. Behaviour of reduction-sorption of chromium (VI) from an aqueous solution on a modified sorbent from rice husk. Water Air Soil Pollut. 163:81-102.

11. FEIZI, M.; JALALI, M. 2015. Removal of heavy metals from aqueous solutions using sunflower, potato, canola and walnut shell residues. J. Taiwan Inst. Chem. Eng. 54:125-136.

12. GUO, Z.; FAN, J.; ZHANG, J.; KANG, Y.; LIU, H.; JIANG, L.; ZHANG, C. 2016. Sorption heavy metal ions by activated carbons with well-developed microporosity and amino groups derived from Phragmites austra- 
lis by ammonium phosphates activation. J. Taiwan Inst. Chem. Eng. 58:290-296.

13. HAMZA, I.A.A.; MARTINCIGH, B.S.; NGILA, J.C.; NYAMORI, V.O. 2013. Adsorption studies of aqueous $\mathrm{Pb}$ (II) onto a sugarcane bagasse/multi-walled carbon nanotube composite. Phys. Chem. Earth. 66:157166.

14. IGWE, J.; ABIA, A. 2006. Sorption Kinetics and Intraparticulate diffusivity of As (III) bioremediation from aqueous solution, using modified and unmodified coconut fiber. Ecl. Quim. Sao Paulo. 31(3): 23-29.

15. INGOLE, N.W.; DHARPAL, S.V. 2012. State of art of biosorption technique for treatment of heavy metals bearing wastes. Int. J. Adv. Eng. Technol. 3(2):143153.

16. ISLAM, S.; AHMED, K.; RAKNUZZAMAN, M.; AL-MAMUN, H.; ISLAM, M.K. 2015. Heavy metal pollution in surface water and sediment: A preliminary assessment of an urban river in a developing country. Ecological indicators. 48:282-291.

17. ISMAIEL, A.A.; AROUA, M.K.; YUSOFF, R. 2013. Palm shell activated carbon impregnated with task-specific ionic-liquids as a novel adsorbent for the removal of mercury from contaminated water. Chem. Eng. J. 225:306-314.

18. LARGITTE, L.; BRUDEY, T.; TANT, T.; COUESPEL DUMESNIL, P.; LODEWYCKX, P. 2016.Comparison of the adsorption of lead by activated carbons from three lignocellulosic precursors. Micropor. Mesopor. Mat. 219:265-275.

19. LAVADO-MEZA, C.; SUN KOU, M.; RECUAY-ARANA, N. 2012. Remoción de $\mathrm{Cr}(\mathrm{VI})$ empleando carbones preparados por activación química a partir de las astillas de eucalipto. Rev. Soc. Quim. 78(1):14-26.

20. LIU, W.; SUN, W.; HANB, Y.; AHMAD, M.; NI, J. 2014. Adsorption of $\mathrm{Cu}$ (II) and $\mathrm{Cd}$ (II) on titanatena nomaterials synthesis via hydrothermal method under different $\mathrm{NaOH}$ concentrations: Role of sodium content. Colloids and Surfaces A: Physicochem. Eng. Aspects.452:138-147.

21. MA, F.; ZHANG, Z.; JIANG, J.; HU, J. 2015. Chromium (VI) potentiates the DNA adducts (O6-methylguanine) formation of $\mathrm{N}$-nitroso dimethyl amine in rat: Implication on carcinogenic risk. Chemosphere. 139:256-259.
22. MÄDLER, S.; SUN, F.; TAT, C.; SUDAKOVA, N.; DROUIN, P.; TOOLEY, R.J.; REINER, E.; SWITZER, T.; DYER, R.; KINGSTON, H.M.S.; PAMUKU, M.; FURDUI, V.I. 2016. Trace-Level Analysis of Hexavalent Chromium in Lake Sediment Samples Using Ion Chromatography Tandem Mass Spectrometry. J. Environ. Prot. 7:422-434.

23. MOMČILOVIĆ, M.; PURENOVIĆ, M.; BOJIĆ, A.; ZARUBICA, A.; RANĐELOVIĆ, M. 2011. Removal of lead (II) ions from aqueous solutions by adsorption onto pine cone activated carbon. Desalination. 276:53-59.

24. OMIDVAR, B.; PIRSAHEB, M.; VOSOUGHI, M.; KHOSRAVI, R.; KAKAVANDI, B.; ZARE, M.; ASADI, A. 2016. Batch and column studies for the adsorption of chromium (VI) on low-cost Hibiscus cannabinus kenaf, a green adsorbent. J. Taiwan Institute of Chemical Engineers. 68:80-89.

25. OWALUDE, S.; TELLA, A. 2016. Removal of hexavalent chromium from aqueous solutions by adsorption on modified groundnut hull. Beni-suef university journal of basic and applied sciences. 5:377-388.

26. OZDES, D.; GUNDOGDU, A.; KEMER, B.; DURAN, C.; KUCUK, M.; SOYLAK, M. 2014. Assessment of kinetics, thermodynamics and equilibrium parameters of $\mathrm{Cr}$ (VI) biosorption onto Pinus brutia Ten. Can. J. Chem. Eng. 92:139-147.

27. SAHA, R.; MUKHERJEE, K.; SAHA, I.; GHOSH, A.; GHOSH, S.; SAHA, B. 2013. Removal of hexavalent chromium from water by adsorption on mosambi (Citrus limetta) peel. Res. Chem. Intermed. 39:224557.

28. SALA, E.; GRANHEN, C.; TAVARES, M. 2005. Biosorption Chromium (III) by Sargassum sp. Biomass. Electron. J. Biotechnol. 5:1-7.

29. TEJADA, C.; QUIÑONEZ, E.; TEJEDA, L.; MARIMÓN, W. 2015a. Absorción de cromo hexavalente en soluciones acuosas por cascaras de naranja (Citrus sinensis). Producción + Limpia. 10(1):9-21.

30. TEJADA, C.; VILLABONA, A.; GARCÉS, L. 2015b. Kinetics of adsorption in mercury removal using cassava (Manhiot esculenta) and lemon (Citrus limonum) wastes modified with citric acid. Ing. Univ. 19(2):283289.

31. TEJADA, C.; VILlabONA, A.; RUIZ, E. 2015c. Cinética de adsorción de $\mathrm{Cr}(\mathrm{VI})$ usando biomasas residuales 
modificadas químicamente en sistemas por lotes y continuo. Rev. ION. 28(1):29-41.

32. TORAB-MOSTAEDI, M.; ASADOLLAHZADEH, M.; HEMMATI, A.; KHOSRAVI, A. 2013. Equilibrium, kinetic, and thermodynamic studies for biosorption of cadmium and nickel on grapefruit peel. J. Taiwan Inst. Chem. Eng. 44(2):295-302.
33. WU, Y.; LUO, H.; WANG, H.; WANG, C.; ZHANG, J.; ZHANG, Z. 2013. Adsorption of hexavalent chromium from aqueous solutions by graphene modified with cetyl trimethyl ammonium bromide. J. Colloid Interface Sci. 394:183-191.

Recibido: Mayo 12 de 2016

Aceptado: Abril 14 de 2017

\section{Cómo citar:}

Tejada Tovar, C.; Villabona Ortiz, A.; Jiménez Villadiego, M. 2017. Remoción de cromo hexavalente sobre residuos de cacao pretratados químicamente. Rev. U.D.C.A Act. \& Div. Cient. 20(1): 139-147. 